\title{
Komplementäre Therapien in den Leitlinien für die Behandlung des Fibromyalgiesyndroms - Update 2012
}

\author{
Romy Lauche Holger Cramer Petra Klose Gustav Dobos Jost Langhorst \\ Lehrstuhl für Naturheilkunde der Alfried Krupp von Bohlen und Halbach-Stiftung, Universität Duisburg-Essen, Deutschland
}

Die aktualisierte Leitlinie zur Definition, Pathophysiologie, Diagnostik und Therapie des Fibromyalgiesyndroms wurde am 17. April 2012 fertiggestellt [1]. Sie entstand unter Leitung von Winfried Häuser von der Deutschen Interdisziplinären Vereinigung für Schmerztherapie und unter Beteiligung von 12 Fachgesellschaften und 2 Selbsthilfevereinigungen (Deutsche Gesellschaft für Neurologie (DGN), Deutsche Gesellschaft für Orthopädie und Orthopädische Chirurgie, (DGOOC), Deutsche Gesellschaft für Physikalische Medizin und Rehabilitation (DGPMR), Deutsche Gesellschaft für Psychiatrie, Psychotherapie und Nervenheilkunde (DGPPN), Deutsche Gesellschaft für Psychologische Schmerztherapie und Schmerzforschung (DGPSF), Deutsche Gesellschaft für Psychosomatische Medizin und ärztliche Psychotherapie (DGPM), Deutsche Gesellschaft für Rheumatologie (DGRh), Deutsche Gesellschaft zum Studium des Schmerzes (DGSS), Deutsches Kollegium für Psychosomatische Medizin (DKPM), Gesellschaft für Kinder- und Jugendrheumatologie (GKJR), Deutsche Gesellschaft für Allgemeinmedizin und Familienmedizin (DEGAM), Zentralverband der Krankengymnasten/Physiotherapeuten (ZVK), Deutsche Fibromyalgie Vereinigung, Deutsche Rheuma-Liga).

Der Bereich zu den komplementären Verfahren wurde dabei federführend von Jost Langhorst, Frauke Musial und Petra Klose ab 2008 in Zusammenarbeit mit der AG komplementäre und alternative Therapieverfahren bearbeitet. Da die Deutsche Gesellschaft für Naturheilkunde (DGNHK, www.gesellschaftnaturheilkunde.de) $\mathrm{zu}$ diesem Zeitpunkt noch nicht als offizielles Mitglied in der Arbeitsgemeinschaft der Wissenschaftlichen Medizinischen Fachgesellschaften e.V. (AWMF) vertreten war, nahmen deren Mitglieder im Rahmen anderer involvierter Fachgesellschaften an der Leitlinienerstellung teil.

Im Rahmen der Version von 2008 wurde eine erste umfassende systematische Literaturrecherche durchgeführt. Hierfür wurden mehrere medizinische Datenbanken wie PubMed, Embase, Scopus und die Cochrane Library durchsucht. Die Recherche basierte auf einer Kombination von Stichworten für Fibromyalgie und komplementäre Verfahren. Für die Suche nach letzteren wurden sowohl allgemeine Begriffe (komplementär, alternativ, integrativ usw.) als auch Bezeichnungen für spezifische Verfahren (Traditionelle Chinesische Medizin, Akupunktur, Balneotherapie, Massage usw.) genutzt. Für die Leitlinie 2012 wurde die Suche um die Studien der Jahre 2008-2012 ergänzt.

Die erste Recherche im Jahr 2008 ergab mehrere tausend Artikel. In aufwendiger Handarbeit wurden relevante Studien zur Behandlung des Fibromyalgiesyndroms identifiziert und die Daten aus diesen Studien extrahiert. Die Datenlage wurde zusammengefasst und in der Konsensuskonferenz allen Beteiligten zur Diskussion und Abstimmung gestellt, woraufhin Empfehlungen zu den einzelnen komplementären Verfahren verfasst wurden. Die Ergebnisse der Recherche zu den komplementären Verfahren wurden zudem in mehreren systematischen Reviews publiziert [2-7].

Insgesamt wurde deutlich, dass für die meisten komplementärmedizinischen Verfahren nur geringe Evidenz existiert. Zudem ist die Qualität der vorhandenen Studien für eine gute Empfehlung innerhalb der Leitlinien oft nicht ausreichend. Die Therapie des Fibromyalgiesyndroms ist nichtsdestotrotz ein Bereich, in dem komplementäre Verfahren bei über $90 \%$ der Patienten eingesetzt werden und somit von hoher klinischer Relevanz sind [1]. In Tabelle 1 sind die Empfehlungen zu komplementären Verfahren aufgelistet. Die wichtigsten Verfahren sind im Folgenden zusammengefasst.

Eine starke Empfehlung mit einem starken Konsens wurde trotz mäßiger Evidenzlage, Wirksamkeit und Akzeptanz für meditative Bewegungstherapien wie Yoga, Qigong und Tai Chi ausgesprochen. Diese Empfehlung basiert auf dem allgemeinen Behandlungsgrundsatz, Patienten zu angemessener

\section{KARGER \\ Fax +497614520714 \\ Information@Karger.de}

www.karger.com (c) 2012 S. Karger GmbH, Freiburg

$1661-4119 / 12 / 0196-0280 \$ 38.00 / 0$

Accessible online at:

www.karger.com/fok
Prof. Dr. med. Jost Langhorst

Klinik für Naturheilkunde und Integrative Medizin

Kliniken Essen-Mitte, Knappschafts-Krankenhaus

Am Deimelsberg 34a, 45276 Essen, Deutschland

j.langhorst@kliniken-essen-mitte.de 


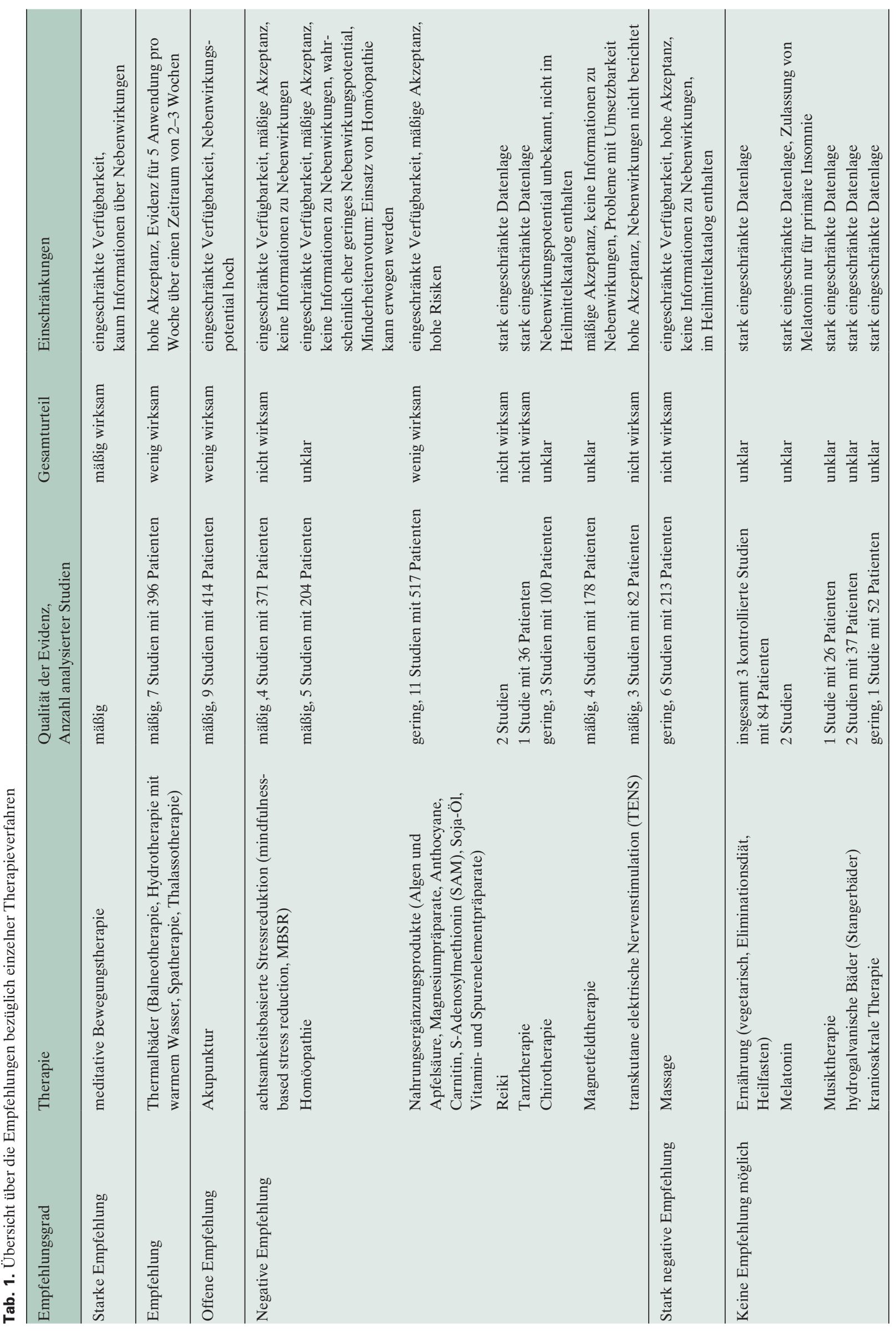


Tab. 2. Veränderungen von Empfehlungsgraden für komplementäre und alternative Verfahren der ersten und zweiten Leitlinienversion im Vergleich

\begin{tabular}{lll}
\hline Therapieverfahren & Empfehlungsgrad 2008 & Empfehlungsgrad 2012 \\
\hline $\begin{array}{l}\text { Achtsamkeitsbasierte Stressreduktion } \\
\text { als Monotherapie }\end{array}$ & keine Aussage & stark negative Empfehlung \\
$\begin{array}{l}\text { Akupunktur } \\
\text { Atemtherapie }\end{array}$ & stark negative Empfehlung & offene Empfehlung \\
Eliminationsdiät & offene Empfehlung & nicht berücksichtigt \\
& offene Empfehlung & keine positive oder negative Empfehlung \\
möglich & keine Aussage \\
Fußzonenreflextherapie & offene Empfehlung & negative Empfehlung \\
Homöopathie & offene Empfehlung & starke Empfehlung \\
Meditative Bewegungstherapien & negative Empfehlung \\
Reiki & keine Aussage & keine positive oder negative Empfehlung \\
Vegetarische Kost / Heilfasten & offene Empfehlung &
\end{tabular}

körperlicher Aktivität zu ermutigen. Es wird jedoch angemerkt, dass die Verfügbarkeit eingeschränkt ist und derartige Verfahren in der Regel nicht von den gesetzlichen Krankenkassen getragen werden.

Eine weitere positive Empfehlung betrifft Thermalbäder, für die trotz mäßiger Evidenz und geringer Wirksamkeit eine hohe Akzeptanz beobachtet wurde. Die vorliegende positive Evidenz wurde bei fast täglicher Anwendung über einen Zeitraum von 2-3 Wochen festgestellt.

Für Akupunktur konnte lediglich eine offene Empfehlung abgegeben werden, d.h. deren Einsatz kann für einen zeitlich befristeten Zeitraum erwogen werden. Die niedrige Evidenz kam vor allem durch die geringe Anzahl von Studien, deren geringe methodologische Qualität sowie potenzielle Nebenwirkungen und eingeschränkte Verfügbarkeit des Verfahrens zustande. Insgesamt zeigte die Analyse der Studien auch bei Akupunktur nur eine geringe Wirksamkeit.

Für alle weiteren Verfahren wurden negative Empfehlungen ausgesprochen bzw. waren aufgrund der unzureichenden Datenlage keine Empfehlungen möglich.

Für achtsamkeitsbasierte Verfahren gab es negative Empfehlungen, wenn diese als Monotherapie eingesetzt werden sollten. In den bisher vorgelegten Studien konnte für deren Wirksamkeit keine ausreichende Evidenz nachgewiesen werden. In der Diskussion wurde allerdings deutlich, dass der Einsatz von achtsamkeitsbasierten Verfahren im Rahmen von multimodalen Konzepten eine wertvolle Bereicherung der Therapiekonzepte darstellen kann. Auch für Massagen ist die aktuelle Evidenzlage negativ, selbst wenn diese von den Patienten gerne genutzt werden.

Gerade im Bereich der komplementären Verfahren gab es im Vergleich zur Vorgängerversion der Leitlinien von 2008 deutlich mehr negative Empfehlungen (Tab. 2). So wurden 2008 sehr viele offene Empfehlungen ausgesprochen, die häufig auf fehlenden Daten basierten und zum Teil ausschließlich auf Wunsch der Vertreter von Selbsthilfeorganisationen in die Leitlinien aufgenommen wurden. Mit dem Update 2012 wurden die Kriterien, die der Bewertung zugrunde liegen, deutlich verschärft.
Für die Homöopathie erfolgte trotz unveränderter Studien- und Evidenzlage eine Abstufung von einer offenen auf eine negative Empfehlung. Dies wurde mit inkonsistenten Studienergebnissen, einer mäßigen Akzeptanz sowie fehlenden Angaben zu Nebenwirkungen begründet, war aber nicht zuletzt der besonderen Situation in der Kommission am Tag der abschließenden Abstimmung über die Homöopathie geschuldet. Vor dem Hintergrund der Tatsache, dass die Leitlinie für das Fibromyalgiesyndrom bisher die erste und einzige AWMF-Leitlinie war, in der die Homöopathie in die Empfehlungen aufgenommen wurde, ist diese Veränderung durchaus von besonderer Bedeutung. Vor diesem Hintergrund wurde von den Vertretern der komplementären Verfahren ein wohlbegründetes Minderheitenvotum verfasst und in die Leitlinie aufgenommen, nach welchem der Einsatz von Homöopathie weiterhin erwogen werden kann.

Insgesamt, so das Fazit der aktuellen Leitlinie, ist die Datenlage bezüglich komplementärer und alternativer Verfahren nicht ausreichend. Dies betrifft zum einen die Anzahl kontrollierter Studien, zum anderen aber auch die Qualität vorhandener Studien. Der Großteil der behandelten Therapieverfahren erhielt keine positive Empfehlung und für einen beträchtlichen Teil konnte keinerlei Empfehlung abgegeben werden. Im Vergleich zu Leitlinien bei anderen Indikationen ist der Bereich komplementärer und alternativer Therapieverfahren allerdings fester Bestandteil in den Leitlinien des Fibromyalgiesyndroms, welches eine der häufigen Indikationen für die ambulante sowie die stationäre naturheilkundliche und multimodal-integrative Therapie darstellt.

Für das Jahr 2013 ist die Aufnahme der DGNHK in die AWMF avisiert, wobei hier Widerstände aus dem Lager konservativer Interessengruppen zu überwinden sind. Mit der Aufnahme wird die DGNHK bei jeder neuen Leitlinie kontaktiert und erhält die Möglichkeit, Vertreter zu entsenden. Aktuell bestehen bereits Kooperationen mit verschiedenen Fachgesellschaften, z.B. der DEGAM oder der Deutschen Gesellschaft für Verdauungs- und Stoffwechselkrankheiten, in deren Leitlinienprojekte die Arbeitsgruppe um Jost Langhorst bereits involviert ist. Weitere Kooperationen sind aus- 
drücklich erwünscht und Impulse hierfür aus dem Netzwerk der Naturheilkunde und Komplementärmedizin sehr willkommen.

Für das Fibromyalgiesyndrom gilt es, aus den Leitlinien neue Impulse für die Forschung z.B. im Bereich ausleitender Verfahren oder stationärer multimodal-integrativer Therapiekonzepte aufzunehmen. Auch die Erforschung der Misteltherapie, mit der es in einigen Zentren klinische Erfahrung gibt, erscheint vielversprechend. Darüber hinaus wären wei- tere Forschungsaktivitäten, die abschließende Klarheit über die Evidenzlage für den Einsatz der Homöopathie beim Fibromyalgiesyndrom bringen, sehr wünschenswert.

\section{Disclosure Statement}

Die Leitlinienarbeit wird durch eine Förderung der Rut- und Klaus-Bahlsen-Stiftung unterstützt.

\section{Literatur}

1 Themenheft «Fibromyalgiesyndrom» - eine interdisziplinäre S3-Leitlinie. Hintergründe und Ziele, Methodenreport, Klassifikation, Pathophysiologie, Behandlungsgrundsätze und verschiedene Therapieverfahren. Schmerz 2012;26:229-348.

2 Langhorst J, Häuser W, Irnich D, Speeck N, Felde E, Winkelmann A, Lucius H, Michalsen A, Musial F: Komplementäre und alternative Verfahren beim Fibromyalgiesyndrom. Schmerz 2008;22:324-333.

3 Langhorst J, Musial F, Klose P, Häuser W: Efficacy of hydrotherapy in fibromyalgia syndrome - a meta-analysis of randomized controlled clinical trials. Rheumatology (Oxford) 2009;48:1155-1159.
4 Baranowsky J, Klose P, Musial F, Häuser W, Dobos G, Langhorst J: Qualitative systemic review of randomized controlled trials on complementary and alternative medicine treatments in fibromyalgia. Rheumatol Int 2009;30:1-21.

5 Langhorst J, Klose P, Musial F, Irnich D, Häuser W: Efficacy of acupuncture in fibromyalgia syndrome - a systematic review with a meta-analysis of controlled clinical trials. Rheumatology (Oxford) $2010 ; 49: 778-788$.
6 Langhorst J, Klose P, Dobos GJ, Bernardy K, Häuser W: Efficacy and safety of meditative movement therapies in fibromyalgia syndrome: a systematic review and meta-analysis of randomized controlled trials. Rheumatol Int 2012. DOI: 10.1007/s00296-012-2360-1.

7 Langhorst J, Häuser W, Bernardy K, Lucius H, Settan M, Winkelmann A, Musial F: Komplementäre und alternative Verfahren beim Fibromyalgiesyndrom. Schmerz 2012;26:311-317. 